\title{
Heterodyne Meter of Transverse and Longitudinal Displacements of Objects
}

\author{
Arkady Arsenyevich Titov, Mikhail Mikhailovich Bakharev \\ Faculty of Instrumentation, Russian Technological University (MIREA), Moscow, Russia \\ Email address: \\ dor7338@mail.ru (A. A. Titov), Bakharev.Mikhail@yandex.ru (M. M. Bakharev)

\section{To cite this article:} \\ Arkady Arsenyevich Titov, Mikhail Mikhailovich Bakharev. Heterodyne Meter of Transverse and Longitudinal Displacements of Objects. \\ World Journal of Applied Physics. Vol. 6, No. 3, 2021, pp. 41-46. doi: 10.11648/j.wjap.20210603.11
}

Received: June 2, 2021; Accepted: June 16, 2021; Published: July 28, 2021

\begin{abstract}
Nowadays, optical methods are widely used to measure the movements of various objects. In this case, it is necessary to measure both longitudinal displacements at a large distance, and transverse ones. Such tasks have to be solved when measuring the displacements of the cutting tools of machine tools. Among the optical methods, the most accurate is the method of heterodyne interferometry. However, this method does not allow making absolute measurements, since the period of the interference pattern is commensurate with the wavelength of light, which requires counting the number of stripes. In addition, the readjustment of this method requires two-frequency lasers and rather complex optical and electronic systems, which significantly complicates their application. To solve this problem, we used the method of heterodyne interferometry developed by the authors, which, in contrast to the known methods, allows us to make absolute measurements of the parameters of objects. This is achieved by creating a period of the interference pattern, which is equal to the speed of sound in the acousto-optical modulator divided by the modulator control frequency. The result was aa block diagram of a device for measuring transverse and longitudinal displacements of objects by the heterodyne method is developed. Analytical expressions are obtained for calculating the signal strength at the photodetector, the periods of interference patterns, the phase shift depending on the transverse and longitudinal displacements, the measurement range and the measurement accuracy, which allowed us to determine the main parameters of the device. To confirm the results obtained, an experiment was carried out. For this, a block diagram of the experiment was developed, with the help of which the influence of the beam divergence on the period of the interference pattern was determined. The experiment showed good agreement between theory and experiment.
\end{abstract}

Keywords: Laser, Acousto-Optic Modulator, Photodetector, Phase Meter, Microprocessor

\section{Introduction}

There are currently various optical methods for measuring the displacements of objects. In new method is proposed to improve the accuracy of interference measurements by isolating the lines of the centers of the interference bandsisophotes [1]. However, this method can only be used when processing interferograms.

A new method for measuring displacements using an optical raster is proposed [2]. The method is easy to implement and has high measurement accuracy. At the same time, the requirements for the accuracy of the raster production are low. The disadvantage of the method is a rather narrow measurement range (up to $1 \mathrm{~m}$.).

The most technically feasible parameters of the surface relief of diffraction gratings are proposed to create the required phase shifts between the diffracted beams and the quadrature modulation signals and to achieve the required accuracy of measurements with nanometric resolution [3]. The disadvantage is the narrow measurement range.

The main issues of constructing a receiving system for a pulsed laser rangefinder with digital signal processing for determining the range are considered and its hardware is described [4]. The disadvantage of the device is limited functionality, since it can not measure transverse movements.

A new fiber-optic converter of micro-displacements of a mirror surface into the power (amplitude) of an optical signal is proposed [5]. The disadvantage is the narrow measuring range.

The results of comparative field tests of rangefinders with 
an eye-safe radiation wavelength of 1.54 microns and a radiation wavelength of 1.06 microns under the same weather conditions are also presented [6]. The disadvantage is the limited functionality of the device.

A new method for determining the astronomical coordinates of an object by finding the direction of the meridian at the observation point and a variant of an optoelectronic device that allows implementing the necessary angular measurements are proposed [7].

The disadvantage is the relatively low measurement accuracy.

A new method of vibration isolation of optical devices is proposed, which significantly improves the accuracy of their measurements [7]. This method can be used in the device we offer.

A new method for determining the distance to cars "in the blind zone" using a side-view camera is proposed [8]. The disadvantage of this method is the relatively low measurement accuracy.

A new mobile high-precision sensor is proposed for measuring the movements and monitoring the geometric parameters of products in production based on the laser triangulation method [9]. The disadvantage of this method is the narrow measurement range.

However, this method can only be used when processing interferograms.

A completely new optical element, the quadrolens, is proposed, which is used as part of a shear speckle interferometer to provide measurements of deformations of objects simultaneously in two mutually perpendicular directions [10]. The disadvantage of the device is a narrow measuring range.

A new method for improving the measurement accuracy by isophotometric processing of the interferogram is proposed [11]. However, this method can only be used when processing interferograms.

Method for measuring the longitudinal movement of an object using a Michelson interferometer is considered [12]. However, to measure the transverse displacements, an additional interferometer is required, which, with this method, does not measure the absolute displacements of the object, so it requires counting the phase cycles. In a heterodyne method for simultaneous measurement of the absolute transverse and longitudinal displacements of the reflector is considered $[13,14]$. The disadvantages include the relatively low accuracy of measuring the longitudinal movements of the object.

The purpose of this article is to develop a high-precision device for measuring the transverse and longitudinal movements of cutting tools of machining machines by the heterodyne method.

\section{Materials and Methods}

The block diagram of the developed device is shown in Figure 1. The peculiarity of this scheme in comparison with the scheme considered is that in order to increase the accuracy of measuring the longitudinal displacements, a zero-order beam from the output of the AOM through the mirror 5 and through the plane-parallel plate 6 is directed to the slit 7, where it interferes with the beams diffracted on the AOM 2. In addition, the scheme includes a collimator 3, which expands the diffracted beams, which allows you to increase the measurement range of transverse displacements.

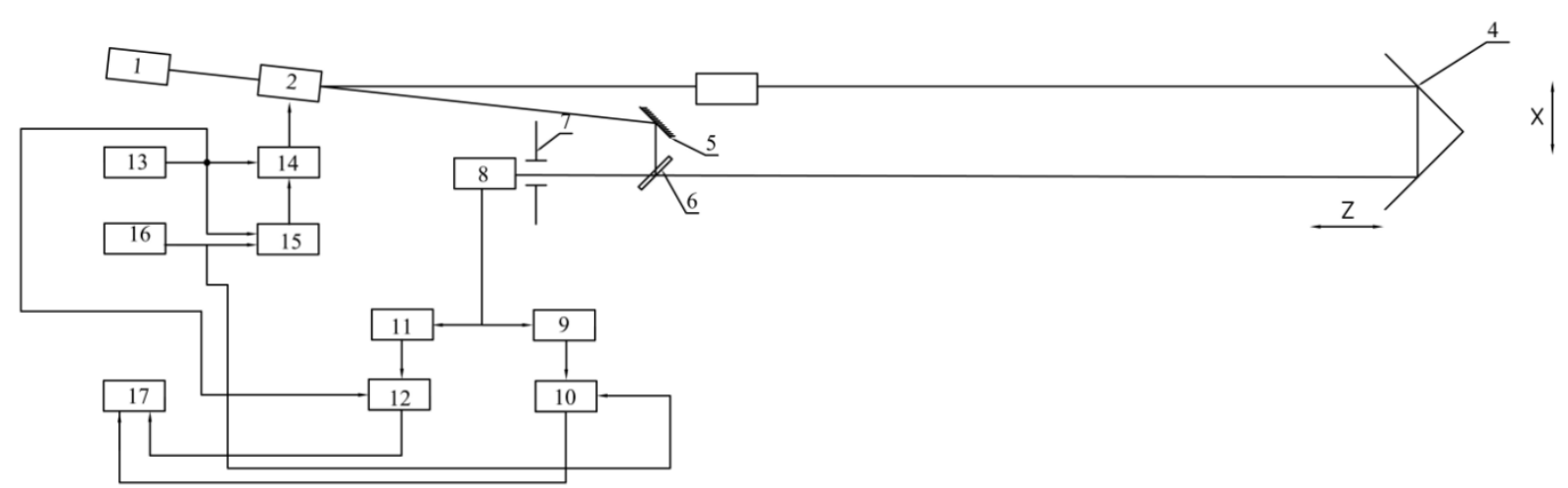

Figure 1. Block diagram of the transverse and longitudinal displacement meter.

1 - laser; 2- acousto-optic modulator (AOM); 3-collimator; 4 -reflector consisting of two mirrors; 5-mirror 6- plane-parallel plate (PPP); 7- slot; 8photodetector; 9-filter low frequencies (LPF); 10-digital phase difference meter; 11-filter high frequencies (HPF); 12-phase discriminator; 13- generator high frequency (GVC); 14 -multiplexer; 15-a phase detector; 16 -low-frequency oscillator (LFO); 17-microprocessor.

With longitudinal movements of the reflector 4 along the $\mathrm{Z}$ axis, the distance between it and the photodetector 8 changes, which leads to a change in the phase shift of the light wave. With the transverse movement of the reflector 4 , the interference pattern created in the beams diffracted on the AOM 2 is shifted along the slit of the photodetector, which also leads to a change in the signal phase. Let us consider in more detail the process of beam interference on the photodetector 8. On the AOM 2, after the multiplexer 14 , the voltages of two frequencies $\mathrm{fb}$ and $\mathrm{fb}+\mathrm{fn}$ are applied, where $\mathrm{fb}$ is the high frequency from the generator 13 , fn is the low frequency from the generator 16. As a result, two diffracted beams are obtained at the output of AOM 2, which interfere with each other. To increase the 
measurement range of transverse displacements, these beams are expanded by the collimator 3 . As a result, when a zero-order beam is fed to the photodetector, interference of three beams will occur. Then the signal at the output of the photodetector, taking into account [15], can be represented in the following form:

$$
\begin{aligned}
& U=A_{0}^{2} S_{\Phi \Pi} l_{u y y} \int_{c-\frac{u x}{2}}^{c+\frac{l_{u x}}{2}} \mid \mathrm{A}_{0} \exp i\left[\left(\omega_{c B}+\omega_{B}\right) t+k L\right]+A_{0} \exp i\left[\left(\omega_{c B}+\omega_{B}+\omega_{H}\right) t+k L \cos \left(\frac{\Theta_{H}}{\beta}\right)+k x \sin \left(\frac{\Theta_{H}}{\beta}\right)\right]+A_{H n} \exp i\left[\omega_{c B} t+\varphi_{o}\right\rfloor d x \\
& \approx 2 A_{0} A_{H n} S_{\Phi \Pi} S_{u} \cos \left(\omega_{6} \mathrm{t}+\mathrm{kL}-\varphi_{o}\right)+2 A_{0} A_{H n} S_{\Phi \Pi} S_{u} \sin c\left(\frac{k l_{u x}}{2 \beta} \Theta_{H}\right) \cos \left[\left(\omega_{6}+\omega_{H}\right) t+k L \cos \frac{\theta_{H}}{\beta}+k c \sin \frac{\theta_{H}}{\beta}-\varphi_{o}\right]+ \\
& 2 A_{0}^{2} S_{\Phi \Pi} S_{u} \sin c\left(\frac{k l_{u x}}{2 \beta} \Theta_{H}\right) \cos \left(\omega_{H} t-\varphi\right)
\end{aligned}
$$

$$
\text { где- } \varphi=-k c \sin \frac{\theta_{H}}{\beta}=-\frac{2 \pi c}{\Lambda_{H} \beta} \text {; }
$$

$$
\Lambda_{н}=\frac{v_{3 в}}{f_{H}}-\text { период интерференционной картинь на выходе АОМ; }
$$

$\mathrm{v}_{\mathrm{zv}}$-the speed of sound in the AOM; - increase in the size of the diffracted beams at the output of the collimator $3 ; \mathrm{A}_{0}$-the amplitude of the diffracted beam at the output of the collimator; $A_{n p}$-the amplitude of the zero-order beam; $S_{\mathrm{sh}}=$ $1 \mathrm{~s}_{\mathrm{hx}}, 1 \mathrm{~s}_{\mathrm{hu}}$ - the dimensions of the gap 7 in the $\mathrm{x}$ and $\mathrm{y}$ coordinates; $\mathrm{S}_{\mathrm{FP}}$-the sensitivity of the photodetector; - the frequency of laser radiation; $\theta_{H}=\lambda f_{H} / v_{3 B} \mathrm{n}_{\mathrm{B}}$ - light diffraction angle; pv-refractive index of air; $k=2 \pi n_{\mathrm{B}} / \lambda$ - wave number; $\lambda$ - the wavelength of the laser radiation; $L$ is the distance from the collimator 3 to the slit 7 .

Filters distinguish signals with frequencies $f_{b}$ and $f_{h}$. Constant phase shift $\varphi_{o}$, introduced by a zero-order beam, it can be compensated by phase shifters. Therefore, we will not take it into account in the future. Then the signals at the output of the filters can be represented as:

$$
\begin{gathered}
U_{в}=2 A_{0} A_{H n} S_{\Phi \Pi} S_{u \xi} \cos \left(\omega_{\theta} t+\frac{2 \pi L}{\lambda}\right) \\
U_{H}=2 A_{0}^{2} S_{\Phi \Pi} S_{u} \sin c\left(\frac{k l_{u x}}{2 \beta} \Theta_{H}\right) \cos \left(\omega_{H} t-\varphi\right)
\end{gathered}
$$

The voltage $U_{v c}$ is applied to the phase discriminator 12, which allocates a proportional signal $\cos \left(\frac{2 \pi L}{\lambda}\right)$ and counts the number of $\mathrm{N}$ phase cycles, which allows the microprocessor 17 to determine the distance L by the expression:

$$
L=N \frac{\lambda}{2}
$$

The voltage Uh is supplied to the digital phase difference meter 10, which measures the phase $\varphi$ and transmits the received information to the microprocessor 17 .

We determine the magnification for the divergent beam. The diameter of the diffracted beam at the collimator output can be represented as:

$$
D=D_{л} \gamma+2 \alpha L / \gamma
$$

where $D_{1}$ is the diameter of the laser beam; - the divergence of the laser; - the apparent magnification of the collimator.

Тогда увеличение размера пучка будет равно:

$$
\beta=\frac{D}{D_{л}}=\gamma+\frac{2 \alpha L}{D_{л} \gamma}
$$

Taking into account (1) and (6), we determine the transverse displacement of the reflector:

$$
C=\frac{\varphi v_{3 B}\left(\gamma+2 \alpha L / D_{л} \gamma\right)}{2 \pi f_{H}}
$$

The microprocessor 17 , using expression 7 , calculates the transverse displacement of the reflector 4.

An important issue in remote measurements of reflector displacements is the determination of the interference region of diffracted beams, since the measurement range of transverse displacements depends on this. An expression was obtained for the interference region of beams, which, in the presence of a collimator, taking into account the divergence of the beam, can be represented as [15]:

$$
h \approx D_{л} \gamma+\frac{2 \alpha L}{\gamma}-\frac{L \theta_{H}}{\left(\gamma+2 \alpha L / D_{л} \gamma\right)}
$$

To determine the measurement range, it is also necessary to determine the power of the signals received by the photodetector. Using the expressions (2), (3), taking into account (5), (6), we obtain formulas for the powers of two harmonics:

$$
P_{в}=\frac{4 P_{\pi} S_{u} \eta(1-\eta) \tau}{\pi\left(\mathrm{D}_{Л} \gamma+2 \alpha L / \gamma\right) D_{л}}
$$




$$
P_{H}=\frac{4 P_{л} S_{u} \eta \tau}{\pi\left(\mathrm{D}_{Л} \gamma+2 \alpha L / \gamma\right)^{2}} \sin c\left(\frac{2 \pi l_{u} f_{\mu}}{v_{3 в}\left(\gamma+2 \alpha L / D_{л} \gamma\right)}\right)
$$

where $\mathrm{Pb}$ and $\mathrm{Ph}$ are the harmonic powers at the frequencies $\mathrm{fb}$ and $\mathrm{fn}$, respectively; - the light loss in the device; - the diffraction efficiency of the AOM; Rl-the laser radiation power.

\section{Results and Discussion}

Let's evaluate the device parameters. When processing large, long parts to control the movement of the cutting tool, the longitudinal and transverse movements can be selected in the range $\mathrm{L}=100 \mathrm{~m}, \mathrm{Cmax}=100 \mathrm{~mm}$.

As a radiation source, we choose a helium neon laser GN 25 with a radiation wavelength of 0.63 microns, a power of $25 \mathrm{MW}$, a beam diameter $\mathrm{Dl}=1.5 \mathrm{~mm}$ and a beam divergence $=1.2 * 10^{-3}$. The coherence length of such lasers reaches $600 \mathrm{~m}$, which will allow the use of this laser in this device. AOM is made on paratellurite, in which the sound propagates at a speed of $617 \mathrm{~m} / \mathrm{s}$ at an excitation frequency $\mathrm{f}_{\mathrm{b}}=15 \mathrm{MHz}$.

Determine the apparent magnification of the collimator $\gamma=\frac{C_{\max }}{D_{л}} \approx 67$. We choose the period of the interference pattern at the output of the AOM equal to the diameter of the laser beam. Then from this condition we can find the low frequency: $f_{H}=\frac{v_{36}}{D_{л}} \approx 0,41 M \Gamma u$. Using the above parameters, using the expression (8), we find the interference region of the beams, therefore, the measurement range of transverse displacements will be preserved over the entire distance. Using expressions (9) and (10), we determine the power of the harmonics. To do this, the light loss and the area of the gap. The diffraction efficiency of the AOM in Bragg diffraction can be 0.9 . The light loss in the device will consist of the losses in the elements (3-6) and will be approximately $\tau=0,3$. In addition, the target function must be taken into account in the expression (10). In order to determine the width of the slit ls, it is necessary to know the size of the photodetector. As a photodetector, we choose a pin photodiode with a threshold sensitivity of $\mathrm{N}=5 * 10-12 \mathrm{~W} *$ $\mathrm{Hz}-0.5$, with a diameter of the photosensitive $\mathrm{V}_{\text {sp }}$ pad $=0.5$ $\mathrm{mm}$. Choose $1_{\mathrm{s}}=\mathrm{Vsp}$. Substituting the values of the parameters defined above in (9-10), we get $R_{v}=10-6 \mathrm{~W}$, $\mathrm{P}_{\mathrm{h}}=10-7 \mathrm{~W}$.

Let us determine how much the values of these powers exceed the minimum radiation power detected by the photodetector, which is determined by the well-known expression: $P_{\min }=G \sqrt{\Delta f}$, where is the frequency band. Then for the used frequencies $\mathrm{f}_{\mathrm{b}}=15 \mathrm{MHz}$ and $\mathrm{f}_{\mathrm{n}}=0.41 \mathrm{MHz}$, respectively, we get $\mathrm{P}_{\min \mathrm{b}}=1.93 * 10-8 \mathrm{~W}, \mathrm{P}_{\min \mathrm{h}}=3 * 10-9 \mathrm{~W}$.

Then we determine the signal-to-noise ratio for these two cases $\left(\frac{S}{N}\right)_{B}=\frac{P_{B}}{P_{\min B}}=51,8 ;\left(\frac{S}{N}\right)_{H}=\frac{P_{H}}{P_{\min H}}=33,3$ this will ensure a sufficiently reliable reading of the information.

We now find the minimum longitudinal and transverse displacements that the device can measure. The minimum longitudinal displacement from (4) will be equal to $\frac{\lambda}{2}=315 \mu$. The minimum transverse displacement can be found from (7), given that the sensitivity of modern digital phase meters is equal to $\varphi=0,01^{0}$. Then from (7) we get $C_{\text {min }} \approx 3$ м $\mathrm{N}$.

Let us determine the relative error $\mathrm{C} / \mathrm{C}$ of the measurement of transverse displacements. To do this, we use the method of differentiating expression (7) by parameters that have errors. Parameters $\alpha, \mathrm{D}_{л}, \gamma, L$ their errors can be measured and taken into account in the course of work, as they are systematic. The remaining parameters are random. Then, given (7), we get:

$$
\Delta X / X \approx \sqrt{(\Delta \varphi / \varphi)^{2}+(\Delta v / v)^{2}+\left(\Delta f_{H} / f_{H}\right)^{2}}
$$

As shown in [15], the specified (11) parameters have the following values: $\Delta \varphi / \varphi=2,7 \cdot 10^{-3}, \Delta v / v=5,2 \cdot 10^{-4}$, $\Delta f_{H} / \Delta f_{H}=10^{-4}$. Substituting these values in (11), we find $\Delta C / C=0,27 \%$.

To test the effect of the laser divergence on the period of the interference pattern, an experimental setup was assembled. The scheme of the experiment is shown in Figure 2. The installation used a semiconductor laser KLM-650/5 with a wavelength of $650 \mathrm{~nm}$, the coherence length of which was sufficient to ensure the interference of closely spaced beams. As a high-frequency generator, the G4-107 generator with amplitude modulation was used.

To create the divergence of the beam, a cylindrical lens 3 is used.

When experimentally determining the period of the interference pattern, the photodetector was first set to the $\mathrm{d} 1$ position and then moved to the $\mathrm{d} 2$ position by a distance L. In both positions, the diameter of the diffracted beam was measured and the phase dependence on the displacement was removed. To do this, the photodetector together with the amplifier was installed on a table, the movement of which was measured by an indicator with a scale division price of 10 microns. An increase in the beam size was determined:

$$
\beta=\frac{d_{2}}{d_{1}}=\frac{40 \mathrm{MM}}{26 \mathrm{MM}}=1,53
$$

where $\mathrm{d} 2$ and $\mathrm{d} 1$ are the beam diameters in two positions.

The table with the photodetector moved perpendicular to the beam with a step of $0.25 \mathrm{~mm}$. The displacement was measured by the ICH - 10 indicator, and the signal phase was measured by the phase difference meter F2-16. The low frequency was $200 \mathrm{kHz}$. The measurement results are shown in the table. 


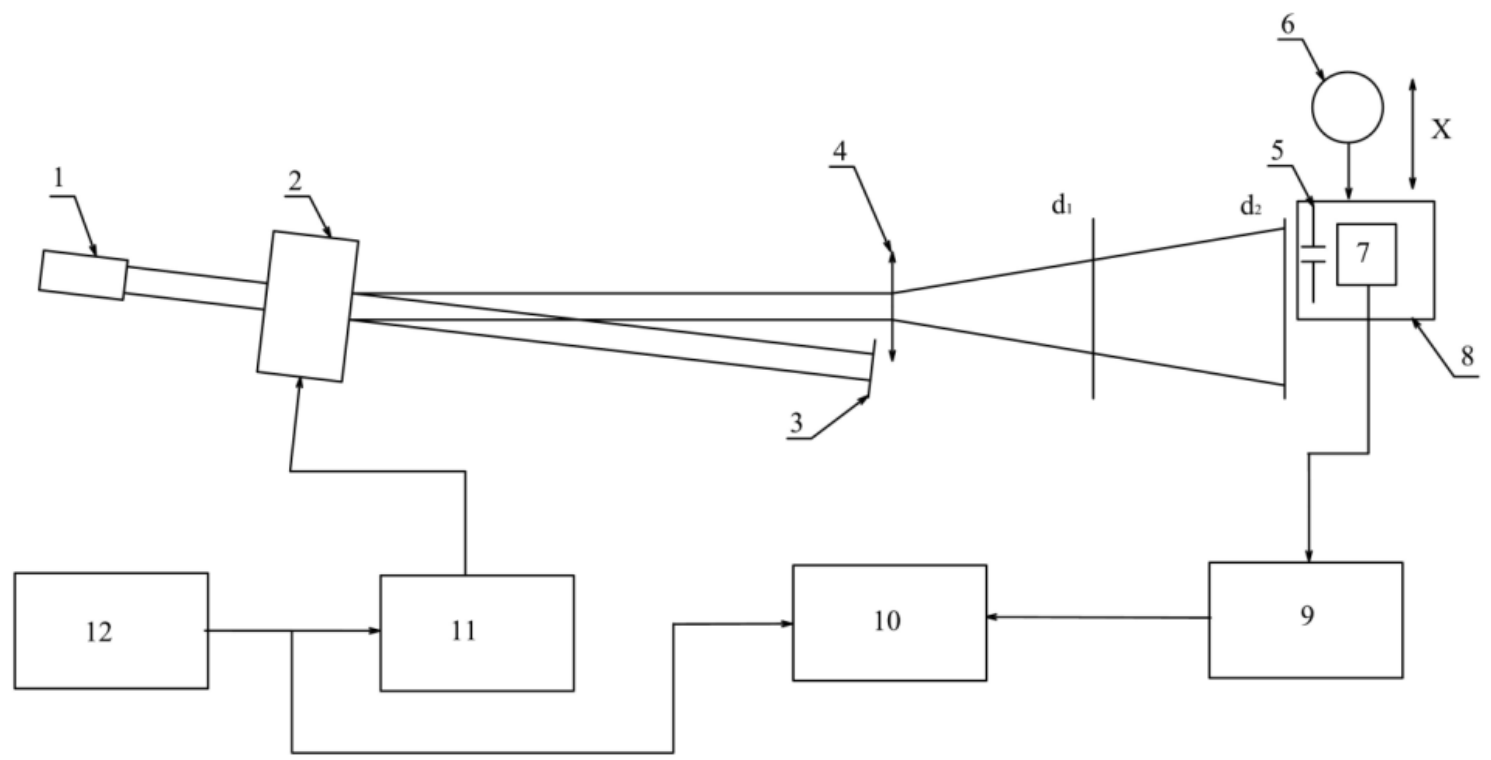

Figure 2. Scheme of the experiment.

1-laser; 2-acousto-optic modulator (AOM); 3-screen; 4-cylindrical lens; 5-slot; 6 - hour indicator; 7-photodetector; 8-movable table; 9-amplifier; 10-phase difference meter; 12-low frequency generator; 11-high frequency generator.

On the basis of the data obtained in Figure 3, graphs are built in Excel.

\section{$\varphi$ (град.)}

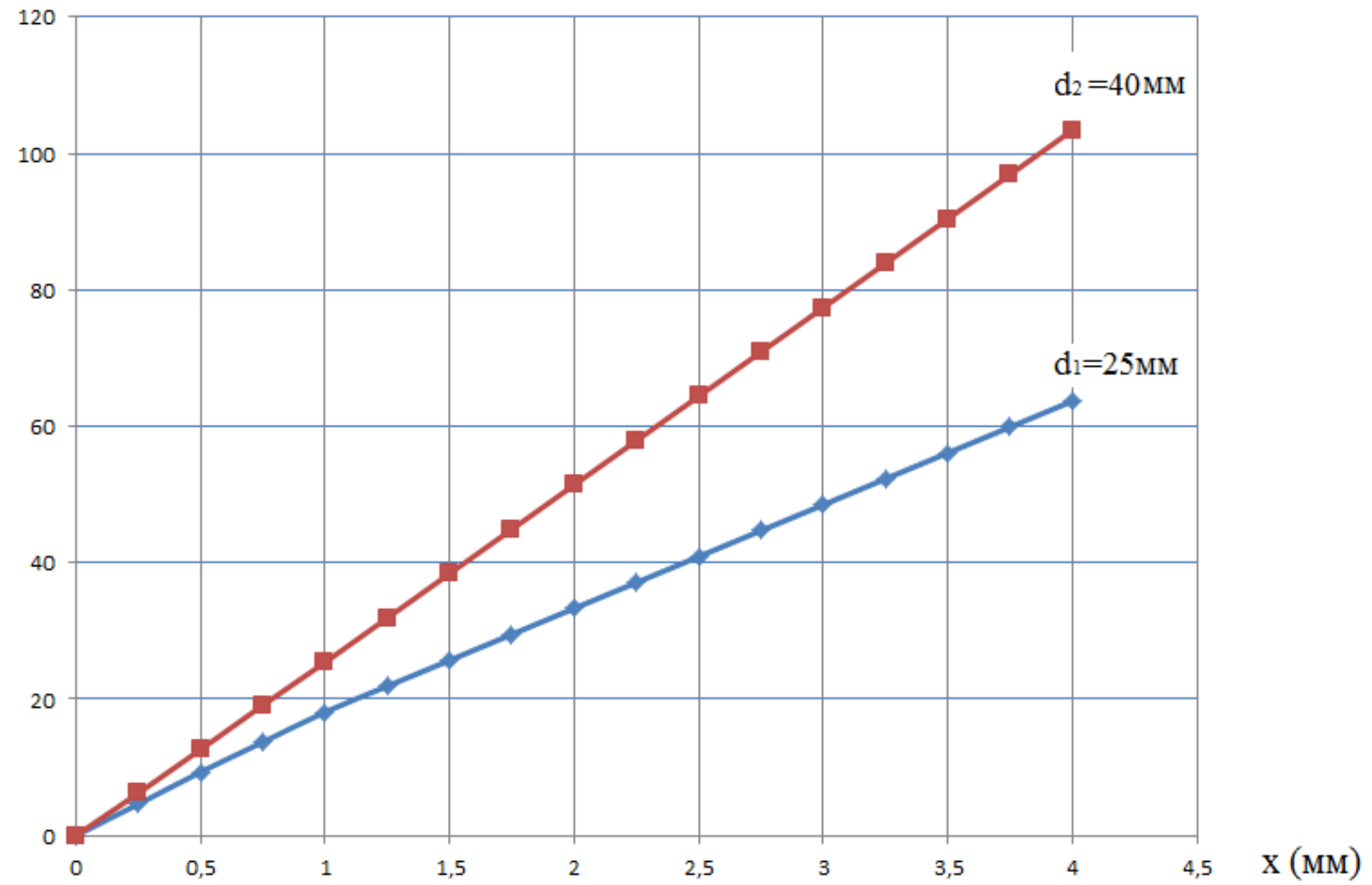

Figure 3. Dependence of the signal phase on the movement of the photodetector at different beam diameters.

We determine the periods of the interference patterns for two beam diameters by the formula:

$$
\Lambda_{1}=\frac{2 \pi \Delta x}{\Delta \phi}=\frac{360^{0} * 1 \mu \mu}{17,3^{0}}=20,8 \text { мM. }
$$




$$
\Lambda_{1}=\frac{2 \pi \Delta x}{\Delta \phi}=\frac{360^{0} * 1 \text { MM }}{25,7^{0}}=14 \mathrm{MM}
$$

where $\Delta x=1 \mathrm{~mm}$ is the selected range of movement of the photodetector; $\Delta \varphi$ is the phase change corresponding to this movement, taken from the table.

Table 1. Dependence of the phase $\varphi$ of the signal on the movement of the table $X$.

\begin{tabular}{lll}
\hline $\mathbf{X}(\mathbf{M м})$ & $\boldsymbol{\varphi}(\mathbf{d e g}$.$) beam diameter \mathbf{d}_{\mathbf{1}}$ & $\boldsymbol{\varphi}(\mathbf{d e g}$.$) beam diameter \mathbf{d}_{\mathbf{2}}$ \\
\hline 0 & 0 & 0 \\
0,25 & 4,6 & 6,2 \\
0,5 & 9,3 & 12,6 \\
0,75 & 13,7 & 19,1 \\
1 & 18,1 & 25,4 \\
1,25 & 21,9 & 31,9 \\
1,5 & 25,7 & 38,4 \\
1,75 & 29,5 & 44,9 \\
2 & 33,3 & 51,4 \\
2,25 & 37,1 & 57,9 \\
2,5 & 40,9 & 64,4 \\
2,75 & 44,7 & 70,9 \\
3 & 48,5 & 77,4 \\
3,25 & 52,3 & 83,9 \\
3,5 & 56,1 & 90,4 \\
3,75 & 59,9 & 96,9 \\
4 & 63,7 & 103,4 \\
\hline
\end{tabular}

The increase in the period of the interference pattern based on the experiment is determined by the expression:

$$
\beta_{\text {эксп. }}=\frac{\Lambda_{2}}{\Lambda_{1}}=\frac{20,8 \text { мM }}{14_{\text {мM }}}=1,485
$$

The error in determining the increase in the beam diameters and in the periods of the interference patterns is equal to:

$$
\Delta \beta=\frac{\beta-\beta_{\text {эксп }}}{\beta_{\text {эксп }}}=4,5 \%
$$

The obtained result indicates a fairly good agreement between the theory and the experiment.

\section{Conclusion}

A block diagram of a device for measuring the transverse and longitudinal movements of an object has been developed. The parameters of the device are estimated and it is shown that the longitudinal movements of the reflector can be measured in the range from $315 \mathrm{~nm}$ to $50 \mathrm{~m}$, and the transverse ones - from 3 microns to $100 \mathrm{~mm}$ with an accuracy of $0.27 \%$. Experimental studies were carried out to assess the effect of the beam divergence on the period of the interference pattern, which confirmed the coincidence of the theory with the experiment.

Thus, the results of the work can be used in the design of a device for measuring the displacements of metal-cutting tools when processing large-sized parts.

\section{References}

[1] Kirillovsky V. K., Tochilina T. V. Application of the computer isophotometric method in interferometry// Izvestiya vuzov priborostroenie. 2021. No 2. pp. 137-142.

[2] Ivanov A. N., Fedorov Yu. V. A method for measuring linear displacements using an optical raster illuminated by a spherical wave front. Izvestiya vuzov priborostroeniya. 2019. No 7. Pp. 654-658.

[3] Odinokov S. B., Shishova M. V., Zherdev A. Yu., Kovalev M. S., Galkin M. L., Venediktov V. Yu. Modeling of phase shifts of light in the orders of diffraction gratings of an interference sensor of linear displacements / / Optics and Spectroscopy, 2019, vol. 127, №. 9, pp. 469-476.

[4] Golovkov V. A., Potapova N. I., Rudenko P. N., Stradov B. G. Reception system of pulsed laser rangefinder / / Optical Journal, 2020, vol. 87, № 11, pp. 74-80.

[5] Vetrov A. A., Sergushichev A. N., Sergushichev K. A. Optimization of the optical scheme of a fiber-optic photometric sensor of micro-displacement, Optical Journal, 2019, vol. 86, №. 4. pp. 45-52.

[6] Moskovchenko L. V., Storoshchuk O. B., Ivanov V. N., Buchenkov V. A. Comparative studies of rangefinders emitting in the micron and one-and-a-half-micron wavelength ranges / / Optical Journal 2019, vol. 86,№ 6. pp. 54-57.

[7] Chudakov Yu. I., Tsvetkov V. I., Azarov S. A. Device for determining the astronomical coordinates of an object // Optical Journal. 2019, Vol. 86. No. 8. pp. 29-35.

[8] Malashin R. O. Measuring the distance to cars using a sideview camera without using road markings / / Optical Journal, 2019, vol. 86, No. 11, pp. 51-58.

[9] Stepanov V. A., Moos E. N., Shadrin M. V., Savin V. N., Umnyashkin A. V., Umnyashkin A. V., Umnyashkin N. V. Triangulation sensor for measuring displacements and highprecision control of product parameters in production. Physical and mathematical sciences. 2020. Vol. 13. No 1. pp. 54-65.

[10] Vishnyakov G. N., Minaev V. L., Ivanov A. D., Vinogradov F. $\mathrm{Yu}$. Shear speckle interferometer with quad-lens//Optics and Spectroscopy, 2020, vol. 128, №10. pp. 1577-1583.

[11] Kirillovsky V. K. Invariance of the principles of isophotometry in computer modeling of interferograms // Izvestiya vuzov priborostroenie. 2021. T. 64. №5. pp. 288-293.

[12] Teleshevsky V. I., Sokolov V. A. Laser measuring information system for increasing the accuracy of multi-axis CNC machines // Vestnik MGTU Stankin. 2011. No. 4. S. 8-10.

[13] Titov A. A., Garipov V. K., Kostromin M. A. Measurements of object displacement by the heterodyne method // Russian technological journal. 2016. No. 1 (10). S. 42-53.

[14] Titov A. A., Amursky V. B., Garipov. V. K. Methods of construction and calculation of laser measuring and storage devices. // M.: Mechanical Engineering. 2008.

[15] A. A. Titov Measurements of Movements of Micro-Objects by the Heterodyne Method // Measurement Techniques. 2017. Vol. 60. No. 2. P. 156-160. 